\title{
Simulation based change detection between DSM and high resolution SAR image
}

\author{
Junyi Tao, Gintautas Palubinskas, Peter Reinartz \\ Remote Sensing Technology Institute \\ German Aerospace Center (DLR) \\ 82234 Oberpfaffenhofen, Germany \\ (Junyi.Tao, Gintautas.Palubinskas, Peter.Reinartz)@dlr.de
}

\begin{abstract}
Change analysis for remote sensing data taken at different times over urban areas provides meaningful information like the expansion of cities. This paper presents a method for the generation of a coregistered geocoded simulated SAR image using a LiDAR digital surface model (DSM) and the comparison to a real SAR image for the purpose of change detection. The DSM and metadata of the SAR image are used for the geocoding of the simulated image, while a line extraction and matching algorithm is used to compensate small shifts between the two images. Afterwards the shadow area in the simulated image is extracted and the corresponding mask is used to analyze the real SAR image in order to detect the changes. An application of the proposed concept is presented for the city centre of Munich where the comparison with the TerraSAR-X GEC product shows a good result. The detected positive changes (new buildings) are compared to a 3D change reference map and show a good correspondence.
\end{abstract}

Keywords: change detection, SAR simulation, line extraction, matching, DSM, TerraSAR-X

\section{INTRODUCTION}

Change analysis for remote sensing data taken at different times over urban areas generates valuable information for analyzing the expansion of the man made structures, or the effect of disasters like earthquakes. Usually the change detection is processed by comparing images taken from the same sensor type (optical or Synthetic Aperture Radar (SAR)) for the two-dimensional case (2D - 2D) [2][3] or by subtracting two digital surface models (DSM) for the three-dimensional case (3D - 3D)[4].

In this paper, we discuss change detection between a DSM as reference with an earlier acquisition data and a high resolution SAR image (3D - 2D). Because of the all-weather and all-time data acquisition capability, SAR images are often the only available data in crisis situations, e.g. directly after an earthquake. For some cities, DSMs have been derived from LiDAR data or high resolution airborne optical image data before the disaster. Change detection between these two data sets may enable a fast analysis of the damage caused by the disaster within the urban area and may lead to an improved rescue management in the disaster area.

The connection between DSM and SAR is established by the SAR simulation technique. Different concepts for SAR simulation are differentiated and classified in [5]. Some work of analysis of the real SAR image using simulation technique are done in [6],[7].

In this paper a method is presented for generation of a coregistered geocoded simulated SAR image using a LiDAR DSM and the comparison and change analysis to a real TerraSAR-X (TSX) image. We use the metadata of TSX to calculate the shift of our geocoded image and use the line extraction and matching algorithm to compensate small shifts between the two images. After that we extract the shadow area in the simulated image and analyze the corresponding mask in the real SAR image to detect the change.

The paper is structured as follows. In section II the method of processing an exactly geocoded simulated image is presented. Section III introduces the change detection method we used. Thereafter the result of the simulated image and the change map is shown in section IV. Finally, the potential and limitations of the method are discussed in section $\mathrm{V}$.

\section{Processing OF GEOCODED SimUlated IMAGE}

\section{A. Generation of a simulated image}

The first step is to process a simulated image using a DSM derived from LiDAR. A SAR simulator named RaySAR [1] is applied, which is developed at the Remote Sensing Technology Institute, Technische Universität München (TUM). A detailed description of the process of SAR simulation using a DSM is presented in [8].

The DSM together with real SAR image acquisition parameters (e.g. azimuth and incidence angle, pixel size) are used as input data for SAR simulation. The result is a simulated SAR image in azimuth and range direction.

\section{B. Geocoding of the simulated image}

The geocoding of the simulated image contains 2 steps. Based on the geoinformation of the DSM, the first step of geocoding is performed as in [8]. The geocoding is the same as in a projection of the simulated image from azimuth-slant range plain onto a horizontal plain with a constant height, which is equal to the minimal height value of the used DSM (in Fig. 1, cyan marked line). 
Some SAR products are also geocoded. For instance, the TerraSAR-X GEC product is represented in map geometry with ellipsoidal corrections. The average terrain height of the respective scene is added to the semi-major and semi-minor axes of WGS84 ellipsoid which are used for correction [10]. The used scene average height can be found in the .xml file. The difference of the used height leads to a constant shift between the simulated image and the real SAR image. The principal is shown in Fig. 1. The red lines indicate the DSM. The black box contains the DSM exactly and is projected onto the cyan marked plain. The cyan dotted line indicates the area of the simulated image. The blue dotted line indicates the area of the real SAR image. We can calculate shifts between them as following:

$$
\begin{gathered}
d E_{1}=(H a-H m) \tan \left(90^{\circ}-i\right) \cos (A z) \\
d N_{1}=-(H a-H m) \tan \left(90^{\circ}-i\right) \sin (A z)
\end{gathered}
$$

Where $d E_{1}$ and $d N_{1}$ are the shifts of the simulated image in East and North direction, $\mathrm{Ha}$ is the scene average height of TSX GEC product, $\mathrm{Hm}$ is the minimum height of the DSM, $A z$ and $i$ are the azimuth and incidence angle.

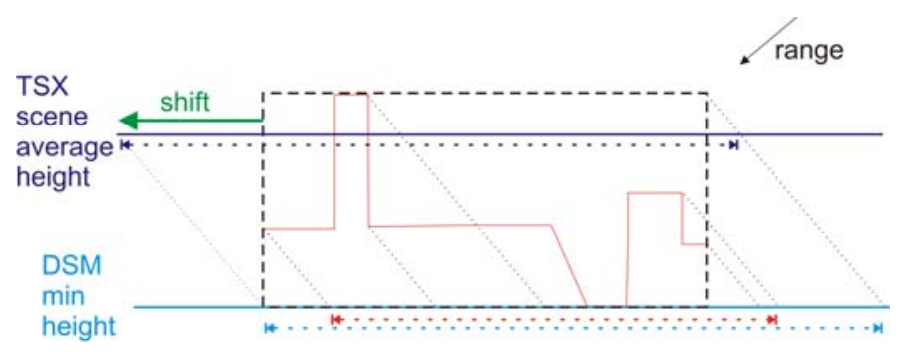

Figure 1. Impact of height difference on geocoding of simulated image.

\section{Lines extraction and matching}

After the geocoding there can still be small shifts between the two images. To solve this problem a matching algorithm is used. The simulator RaySAR uses the geometry of the SAR image, thus a feature based matching algorithm is suitable for our case. We use a function in software Halcon [11] to extract lines from the real SAR image and match the long lines to the simulated image. A detailed description of the line extraction and matching is shown in [9]. For the output we obtain three parameters: the shift in row and column direction, and the rotation angle. In our case, the rotation angle should be zero.

\section{Shift of the simulated image using the matching result}

After the matching, the geocoding of the simulated image will be changed as following:

$$
\begin{gathered}
d E_{2}=- \text { Column }^{*} P s \\
d N_{2}=\text { Row }^{*} P s
\end{gathered}
$$

Where $d E_{2}$ and $d N_{2}$ are the second shift of the simulated image in East and North direction, Row and Column are the output of the matching result, $P s$ is the pixel size of the image.

The simulated image can then be overlaid with the real SAR image exactly. Since the border of the simulated image contains no meaningful values, this would influence the change detection step. Therefore both images will be cut to represent the same range of columns and rows.

\section{CHANGE DETECTION}

After the processing described in part II, we obtain two equal-sized coregistered geocoded images. By the simulation we have converted the $3 \mathrm{D}-2 \mathrm{D}$ change detection problem to $2 \mathrm{D}$ $2 \mathrm{D}$ issue. However, we can not use the typical 2D change detection method like image differencing or ratioing in our case, because the simulated image exhibits no real radiometric signatures of the objects in a scene. But we can still use the geometrical meaning of the grey values in the simulated image. One of the interesting geometrical features is the shadow.

Because of the slanted acquisition geometry of the SAR sensor, shadowed areas occur in the SAR image very often, especially in images of urban areas. The shadow areas in the simulated image contain no signal, i.e. the grey value for shadow is zero. For the same area in the SAR image, the intensity should be very low, in comparison to other pixels. If some new buildings are built in or near the "shadow area" between the acquisition time of the DSM and SAR, there will be high intensity of pixels in the "shadow area". Through detection of these pixels we can find the positive changes.

The detailed steps of the change detection algorithm are described as following:

- $\quad$ Find the shadow mask in the simulated image, where the intensity equals zero.

- Use the mask in the real SAR image, extract the intensity in the mask, convert the intensity to ln(intensity) (logarithmic intensity), plot a logarithmic histogram.

- Because the logarithmic histogram shows a normal distribution, we can calculate the mean value and standard deviation of the $\ln ($ intensity).

- The area where $(\ln ($ intensity $)<$ mean $+2 *$ std $)$ is considered to be a shadow in the SAR image, other pixels are considered as a change.

- Extract the change mask.

- A median filter is used to reduce the noise. The result is a change map.

\section{EXPERIMENTAL RESULTS}

For the simulation we use a DSM of Munich city center with a horizontal and vertical resolution of 1 meter and 0.1 meter, respectively. The DSM is generated from a LiDAR point cloud data acquired in February 2003 (Fig. 2).

For this case study, we use the orthorectified TerraSAR-X (TSX) image (GEC product) (Fig. 3), having a pixel size of 0.5 meter, which has been captured on a descending orbit with an incidence angle of $49.9^{\circ}$ on 7 June 2008. 


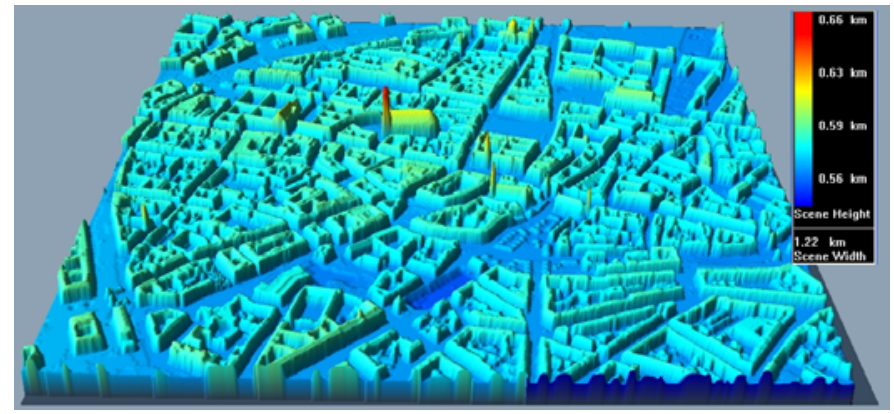

Figure 2. Digital surface model of Munich centre, $1200 \mathrm{~m} * 1200 \mathrm{~m}$.

\section{A. Result of the geocoded simulated image}

Using the geoinformation of the DSM, the simulated image (Fig. 3) is projected to the plain with a height of the minimal value in the DSM. Using the equations (1)-(4), the shift of the simulated image to the real SAR image is calculated and shown in Table I. After the use of the scene average height in the metadata of the TSX GEC product, the shift of the line matching is very low, but still important to correct the data.
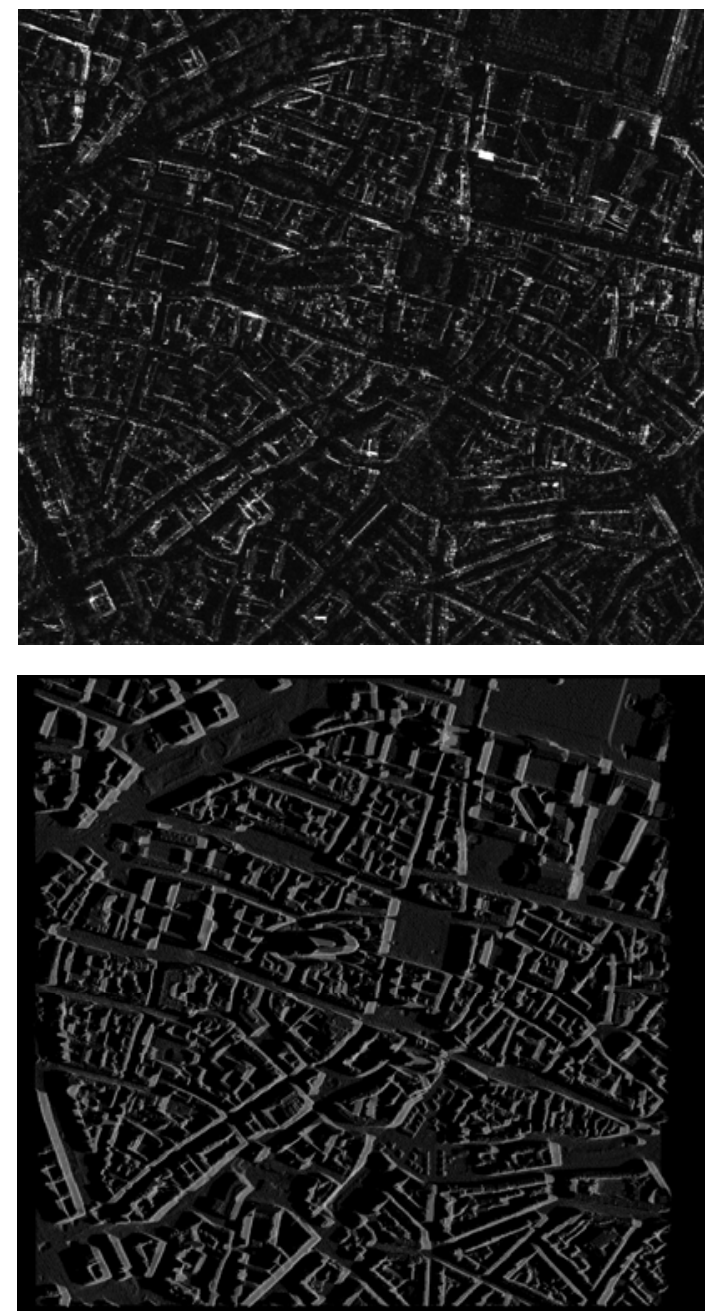

Figure 3. TSX GEC product (upper image), and the coregistered geocoded simulated image (lower image)
TABLE I. SHIFT OF THE SIMULATED IMAGE

\begin{tabular}{|l|c|c|}
\hline Reason & $\mathbf{d E}[\mathbf{m}]$ & $\mathbf{d N}$ [m] \\
\hline $\begin{array}{l}\text { Height difference of the } \\
\text { projection plain }\end{array}$ & -44.50 & 6.53 \\
\hline Line matching & 5.43 & -0.70 \\
\hline
\end{tabular}

\section{B. Result of the change detection}

The shadow area of the simulated image is shown in Fig.4. $46 \%$ pixels in the image have a value 0 .

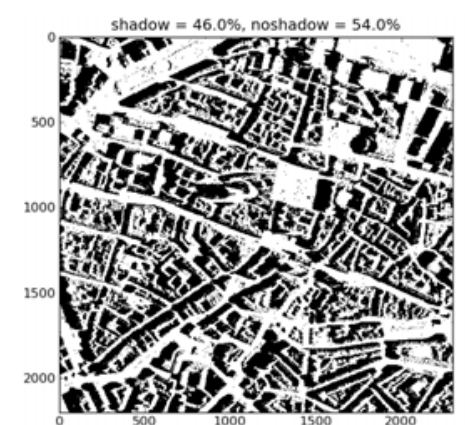

Figure 4. Shadow area (black) in the simulated image.

The shadow mask of the simulated image is used to extract the same pixel from the TSX image. Fig. 5 shows the logarithmical intensity of the "shadow area" in the TSX image. All the pixel values of the "no shadow" area are changed to zero (dark blue). The histogram can be approximated by a normal distribution.

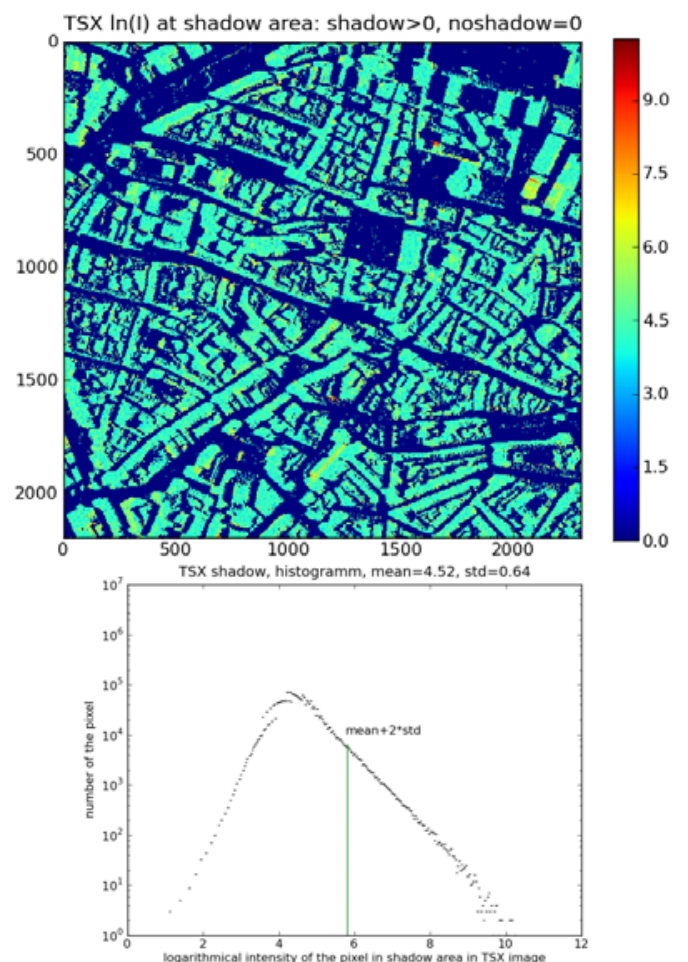

Figure 5. Analysis of the "shadow area" of the TSX image. Upper image: colour indicate the logarithmical intensity of the "shadow area" in TSX image, dark blue colour indicate the "no shadow area". Lower image: logarithmical histogram of the upper image. 
All pixels with $\ln ($ intensity) smaller than (mean+2*standard deviation) are considered as "real shadow", they are eliminated and the result is shown in Fig. 6.

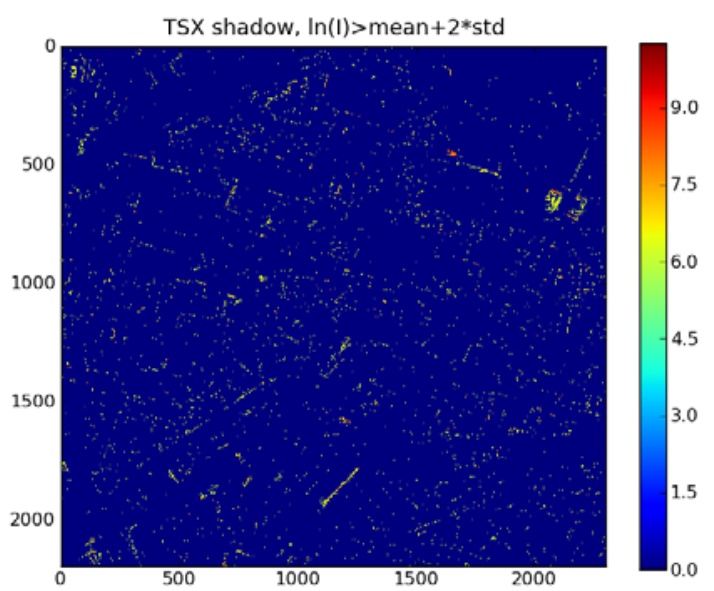

Figure 6. TSX image of shadow area after thresholding.

After using a median filter the noise is reduced. The result is considered as change map and compared with the 3D change detection result in [4]. Both images are shown in Fig. 7. Three from five buildings are detected with our method.
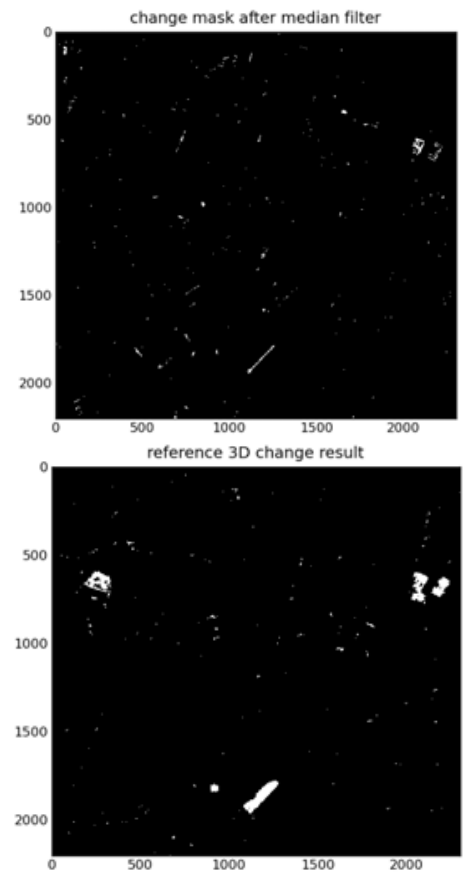

Figure 7. Change maps of our result (upper image) compared to the change map of the 3D change detection result in [4] (lower image).

\section{CONCLUSION}

In this paper, a method for generating a geocoded simulated SAR image using a LiDAR DSM and the comparison and change analysis to a real SAR image is presented. Based on a previous work [8], the geocoding is improved by using the scene average height of TSX GEC product. Small shifts are compensated based on the line extraction and matching algorithms. The shadow area of the simulated image is extracted and the corresponding mask in the real SAR image is analyzed. The high intensity pixels in the mask are extracted using statistical threshold, filtered using median filter and finally considered as positive changes between the acquisition time of the DSM and SAR. The result is compared to a 3D change reference map and shows good correspondence.

Using this method we can obtain the position of the positive change (e.g. new buildings) near the shadow of other buildings, which occur very often in the urban area. A detailed analysis of the change type and size is still needed. The exactly coregistered simulated image presents a good pre-requisite for further change analysis.

\section{ACKNOWLEDGMENT}

TerraSAR-X data were provided by DLR through the Science Projects MTH0505 and MTH0948. The work of Junyi Tao was supported by the DLR-DAAD research grant A/09/95888.

\section{REFERENCES}

[1] S. Auer, S. Hinz, R. Bamler, "Ray-tracing simulation techniques for understanding high-resolution SAR images," IEEE Transactions on Geoscience and Remoe Sensing, vol. 48, no.3, pp. 1445-1456, March 2010.

[2] A. SINGH, "Digital change detection techniques using remotely-sensed data,” International Journal of Remote Sensing, vol. 10, no.6, pp. 9891003, 1989.

[3] D. Lu, P. MAUSEL, E. BRONDIZIO and E. MORAN, "Change detection techniques,” International Journal of Remote Sensing, vol. 25, no.12, pp. 2365-2407, 2004.

[4] J. Tian, H. Chaabouni-chouayakh, P. Reinartz, T. Krauss and P. d'Angelo, "Automatic 3D change detection based on optical satellite stereo imagery,” In: IntArchPhRS, vol. 38, part 7B, pp. 586-591. ISPRS Technical Commision VII Symposium - 100 Years ISPRS, 5.-7. Jul. 2010, Vienna, Austria.

[5] T. Balz, "SAR simulation of urban areas: techniques and applications," In book: Radar Remote Sensing of Urban Areas, Remote Sensing and Digital Image Processing, Springer Science, pp. 215-231, 2010.

[6] T. Balz. "SAR simulation based change detection with high-resolution SAR images in urban environments," In: IAPRS, vol. 35, Part B, Istanbul, 2004.

[7] D. Brunner, G. Lemoine, L. Bruzzone, and H. Greidanus, "Building height retrieval from VHR SAR imagery based on an iterative simulation and matching technique," IEEE Transaction on Geoscience and Remote Sensing, vol. 48, no. 3, pp. 1487-1504, Mar. 2010.

[8] J. Tao, G. Palubinskas, P. Reinartz, S. Auer, "Interpretation of SAR images in urban areas using simulated optical and radar images," 2011 Joint Urban Remote Sensing Event (JURSE), pp. 41-44, 11-13 April 2011.

[9] J. Tao, G. Palubinskas, P. Reinartz, “Automatic interpretation of high resolution SAR images: first results of SAR image simulation for single buildings," in Proc. of ISPRS Hannover Workshop, 14-17. June, 2011, Hannover, Germany.

[10] H. Breit, T. Fritz, U. Balss, M. Lachaise, A. Niedermeier, M. Vonavka, "TerraSAR-X SAR processing and products," IEEE Transactions on Geoscience and Remote Sensing, vol.48, no.2, pp. 727-740, 2010.

[11] Halcon reference manual version 10.0.1. http://www.mvtec.com/download/reference (accessed 30 Jun. 2011). 